\title{
The microcalcification influence on the ateroma plaque vulnerability
}

\author{
M. Cilla, E. Peña, M.Á. Martínez \\ Applied Mechanics and Bioengineering (AMB) \\ Instituto de Investigación en Ingeniería de Aragón (I3A) \\ Universidad de Zaragoza, Mariano Esquillor s/n, 50018, Zaragoza, Spain. \\ Tel.+34-976762707, Fax +34-976762043, e-mail: mcilla@unizar.es
}

\begin{abstract}
Rupture of atherosclerotic plaque, which is related to maximal stress conditions in the plaque, is a major cause of mortality worldwide. More careful examination of stress distributions in plaques reveals that it may be caused by the local stress behaviors caused by cap thinning, inflammation, macroscopic heterogeneity, and recently, the presence of microcalcifications, at critical sites. However, the role of microcalcifications is not yet fully understood and most finite element models of blood vessels with atheroma plaque do not consider the heterogeneity of the plaque constituents at the micro-scale. The goal of the present work is to investigate the effects of microcalcifications on the stress field of an atheroma plaque vessel section. This is achieved by performing a parametric finite element study, assuming a plane strain hypothesis, of an idealized coronary artery cross-section with eccentric atheroma plaque and one microcalcification incorporated. The model was extruded at a small length in order to introduce some purely 3D parameters such as the fiber orientation. Healthy (adventitia) and diseased (fibrotic, lipidic and calcification) tissues were considered. The parametric analysis includes the most influential geometric parameter on atheroma vulnerability and the position and dimensions of the microcalcification: the fibrous cap thickness, the microcalcification ratio, angle and eccentricity. In total, a large set of 108 different geometries were studied. We could conclude that microcalcifications should be considered in the modeling of this kind of problems since they cause a significant alteration of the vulnerable risk by increasing the maximum maximal principal stress up to $40 \%$.
\end{abstract}
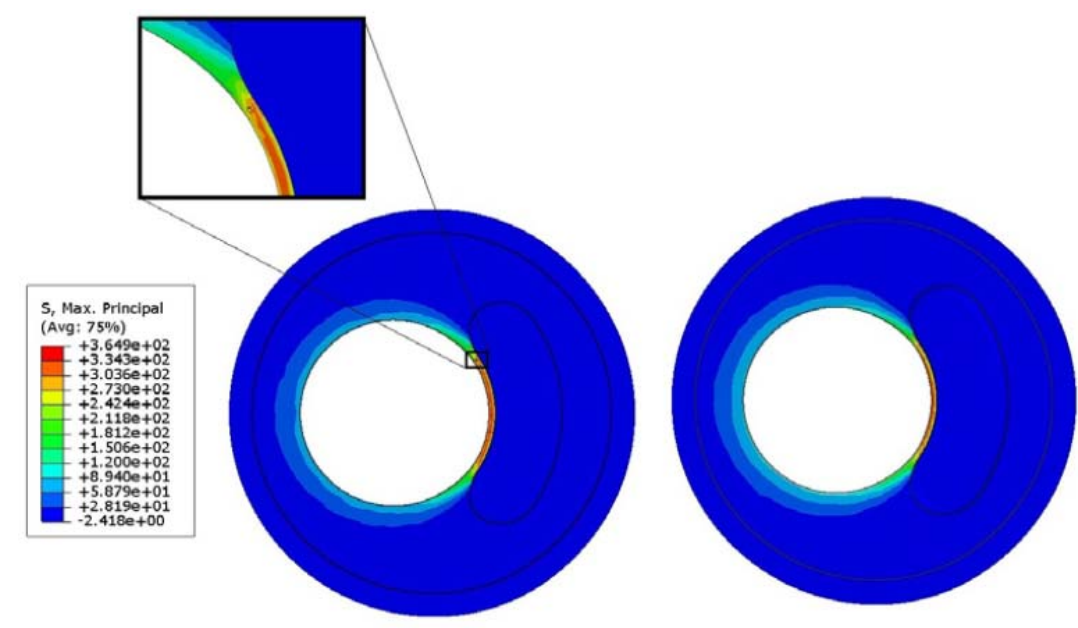

Contour maps of MPS. Comparison between models with and without microcalcification 